\title{
Impact of mevalonate kinase deficiency (MKD) on the quality of life in children and young adults: a national multicentre study
}

\author{
Silvia Federici ${ }^{*}$, Alberto Tomasini ${ }^{2}$, Antonella Meini ${ }^{3}$, Matteo Doglio ${ }^{1}, \mathrm{G}$ Calcagno ${ }^{4}$, Francesco Zulian ${ }^{5}$, \\ Rita Consolini ${ }^{6}$, Martina Finetti ${ }^{1}$, Luciana Breda ${ }^{7}$, Roberta Caorsi ${ }^{1}$, Laura Obici ${ }^{8}$, Romina Gallizzi ${ }^{9}$, Donato Rigante ${ }^{10}$, \\ Mariolina Alessio ${ }^{11}$, Alberto Martini ${ }^{1}$, Marco Gattorno ${ }^{1}$
}

From 18th Pediatric Rheumatology European Society (PReS) Congress

Bruges, Belgium. 14-18 September 2011

\section{Background}

MKD is an autosomal recessive disease caused by mutations in the mevalonate kinase (MVK) gene.

\section{Aim}

To analyze the long term follow-up and health related quality of life (HRQL) in MKD.

\section{Methods}

MVK gene was analyzed in 950 consecutive patients with periodic fever. $40 \mathrm{MKD}$ patients were identified. Spontaneous disease course was classified as follows: i) resolution (no episodes in the last 6 months), ii) improvement (reduction of more then $30 \%$ of fever episodes) iii) stationary iv) worsening (increase frequency of fever episodes or appearance of new major clinical manifestation).The Child Health Questionnaire (CHQPF 50) was used to assess the health related quality of life (HRQL). An international sample of 3315 healthy children (52.2\% female), with a mean (SD) age of 11.2 (3.8) years constituted the healthy control group.

\section{Results}

Data on follow-up are available for 31 patients. The mean follow-up was 12.9 years (range 2.3-38.2). Steroid on demand was effective in treating fever episodes. 15 patients showed a significant spontaneous reduction of the frequency of fever episodes. Complete resolution was observed in 3 patients. In 9 patients the disease was stable, in 4 worsened. When compared to healthy age- matched individuals, HRQL of MKD patients was generally affected, particularly for global health, general health perception, mental health, parental-impact emotion and self-esteem $(p<0.001)$.

\section{Conclusions}

Even if a relevant percentage of MKD patient show a spontaneous amelioration of the disease, most of them display a tendency towards a persistence of fever episodes that significantly affect their quality of life.

\section{Author details}

${ }^{1}$ Gaslini Institute, Genova, Italy. ${ }^{2}$ IRCCS Burlo Garofolo, Dipartimento di Pediatria, University of Trieste, Trieste, Italy. ${ }^{3}$ Dipartimento di Pediatria, Unità di Immunologia e Reumatologia Pediatrica, Spedali Civili E University Of Brescia, Italy. ${ }^{4}$ Sezione di Reumatologia Pediatrica, AOU "G. Martino ${ }^{\prime \prime}$ Messina, Italy. ${ }^{5}$ Dipartimento A.I. di Pediatria,University of Padua, Padova, Italy. ${ }^{6}$ Dipartimento di Medicina della Procreazione e dell'Eta' Evolutiva, Pisa, Italy. ${ }^{7}$ Clinica Pediatrica,Divisione reumatologia, Ospedale Policlinico di Chieti, Chieti, Italy. ${ }^{8}$ Laboratorio di Biotecnologie, IRCCS Policlinico San Matteo, Pavia, Italy. ${ }^{9}$ Divisione di Immunologia e Reumatologia Pediatrica,università di Messina, Messina, Italy. ${ }^{10}$ Università Cattolica del Sacro Cuore, Roma, Italy.

${ }^{11}$ Dipartimento di Pediatria, Università Federico II, Napoli, Italy.

Published: 14 September 2011

\section{doi:10.1186/1546-0096-9-S1-P24}

Cite this article as: Federici et al.: Impact of mevalonate kinase deficiency (MKD) on the quality of life in children and young adults: a national multicentre study. Pediatric Rheumatology 2011 9(Suppl 1):P24.

${ }^{1}$ Gaslini Institute, Genova, Italy

Full list of author information is available at the end of the article

(c) 2011 Federici et al; licensee BioMed Central Ltd. This is an open access article distributed under the terms of the Creative Commons Attribution License (http://creativecommons.org/licenses/by/2.0), which permits unrestricted use, distribution, and reproduction in any medium, provided the original work is properly cited. 